\title{
Facile synthesis of biologically important indole based quinoxalines
}

\author{
Sukanta Kamila, Haribabu Ankati, and Edward R. Biehl* \\ Department of Chemistry, Southern Methodist University, 3215 Daniel Avenue, \\ Dallas, TX-75275, USA \\ E-mail: ebiehl@smu.edu
}

\begin{abstract}
Condensation of 1,2-phenylenediamine with a variety of indole based aldehydes, prepared from the corresponding acid chloride in presence of $\mathrm{HSnBu}_{3}$, furnishes (1H-indol-3-yl)quinoxalines. In addition, 1,2-phenylenediamines substituted with a strong electron-withdrawing group at the para position, provides 6-substituted (1H-indol-3-yl)quinoxalines. Several biologically important quinoxalines were prepared in the same way. The yields are good to excellent in all cases. However, 1,2-phenylenediamine substituted with the weakly electron-donating methyl group, gives an inseparable mixture of 6-methyl and 7-methyl isomers of (1H-indol-3-yl)quinoxaline. All the compounds were characterized by ${ }^{1} \mathrm{H}$ NMR, ${ }^{13} \mathrm{C}$ NMR and IR spectroscopy.
\end{abstract}

Keywords: Oxoacetaldehyde, quinoxalines, indole, oxoacetyl chloride

\section{Introduction}

Numerous quinoxaline derivatives have important biological activity such as antibacterial, antifungal, anticancer, antidepressant and anti-inflammatory agents. ${ }^{1-3}$ Several groups have reported on the biological effects of "plated-derived-growth-factor" (PDGF) tyrosin kinase blockers from the indole-containing blockers, ${ }^{4}$ quinoxaline blockers. ${ }^{5,6}$ In addition, some piperazinylquinoxalines behave as $5-\mathrm{HT}_{3}$ receptor antagonists. ${ }^{7 \mathrm{a}}$

The quinoxaline antibiotics of octadepsipeptide type, e.g., echinomycin (Figure 1), show activity against gram-positive bacteria and certain animal tumors and also are potent inhibitors of RNA synthesis. ${ }^{7 b}$ Some of the marine sponge bis(indole) alkaloids of the topsentin class (Figure 1) have received considerable attention because of their potent biological properties such as antitumor, antiviral, and anti-inflammatory activities. ${ }^{7 \mathrm{c}}$ Consequently, we decided to synthesize some indole based quinoxaline derivatives with the aim of investigating their antimicrobial and neuroprotecting properties. In this present study we report the synthesis of several indole based quinoxalines. 


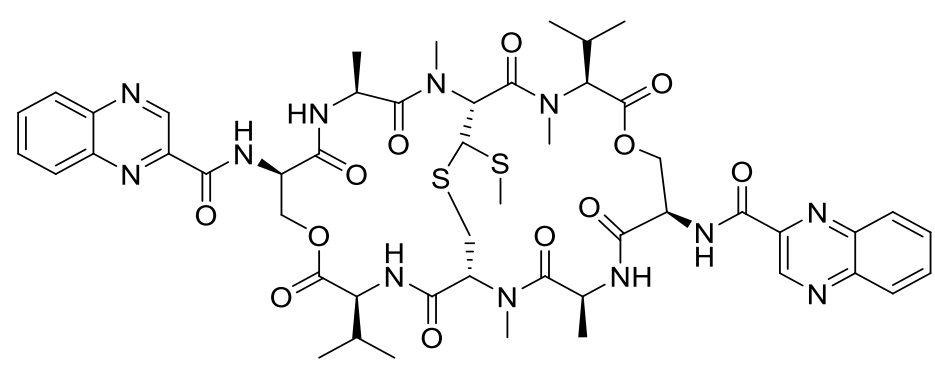

Echinomycin

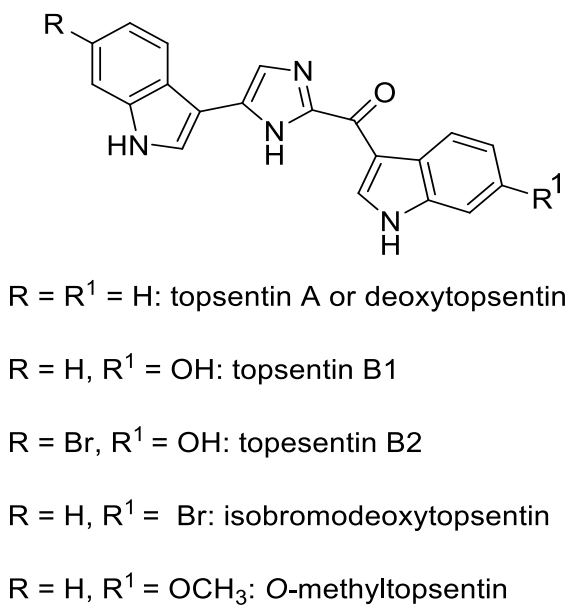

Figure 1

\section{Results and Discussion}

The quinoxalines 4a-p were prepared according to Scheme-1. 3-Indolyl- $\alpha$-oxoacetyl chloride derivatives 2a-f were first prepared by the reaction of corresponding indoles with oxalyl chloride in ether. ${ }^{8}$ All the acid chlorides were isolated and characterized by ${ }^{1} \mathrm{H}$ NMR, ${ }^{13} \mathrm{C}$ NMR and IR spectroscopy. Some of the acid chlorides were previously reported. ${ }^{9,10}$ Treatment with $\mathrm{Bu}_{3} \mathrm{SnH}$ in ethyl acetate gave the corresponding aldehyde intermediates ${ }^{11}$ which because of their instability were immediately treated with suitably substituted 1,2-phenylenediamines 3a-f in presence of base to afford the expected ( $1 H$-indol-3-yl)quinoxalines 4a-p. We studied the reaction in different bases and solvents with 2-(1H-indol-3-yl)-2-oxoacetaldehyde (Entry 1, Table 2) and 1,2phenylenediamine 3a and found that piperidine-ethanol combination gave the best yields of $(1 \mathrm{H}$ indol-3-yl)quinoxaline 4a. The results are summarized in Table 1.

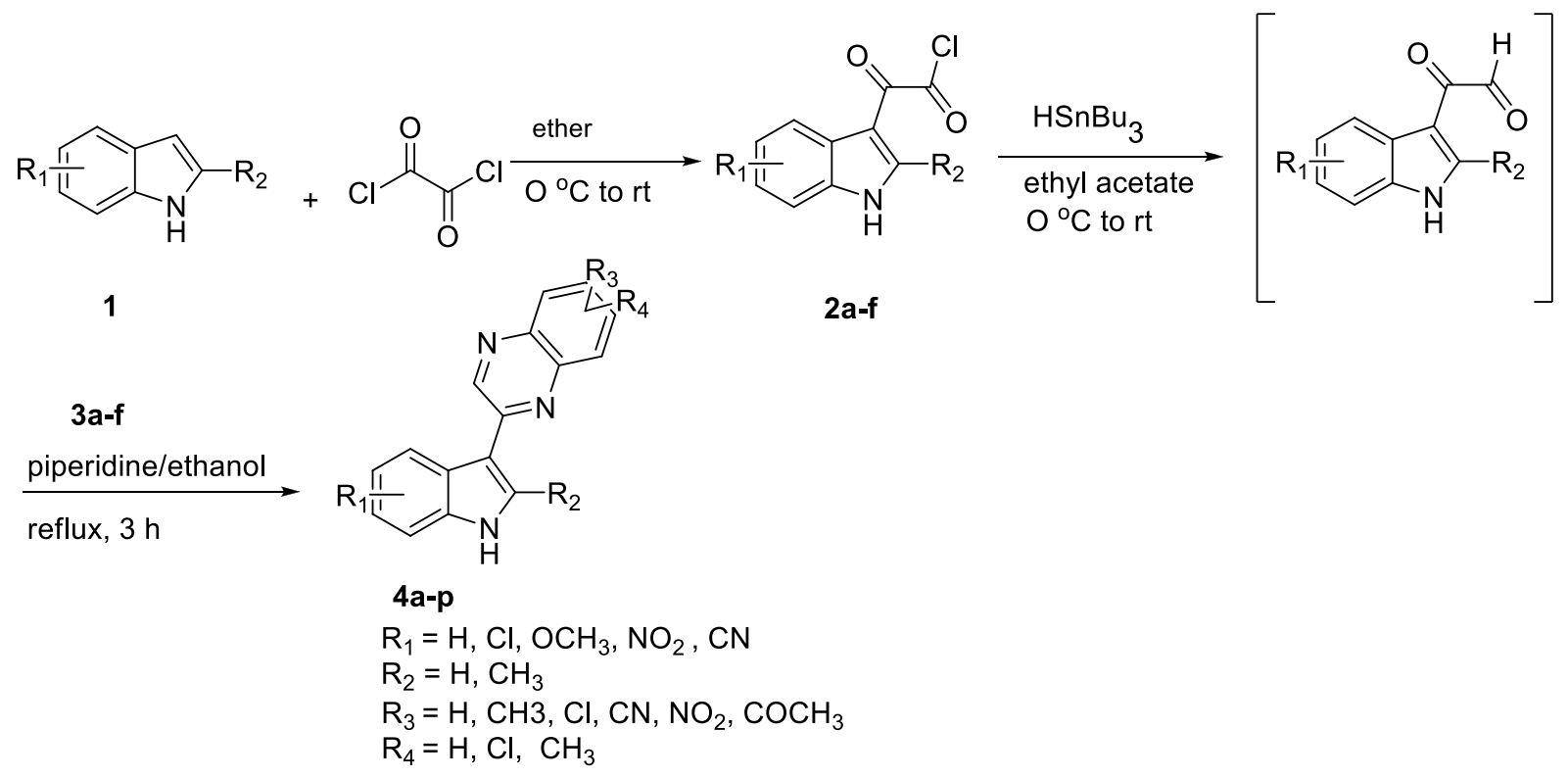

Scheme 1. Schematic representation for the synthesis of indole based quinoxalines. 
Table 1. Reaction of 2-(1H-indol-3-yl)-2-oxoacetaldehyde with 1,2-phenylenediamine in different solvents and bases at $90{ }^{\circ} \mathrm{C}$

\begin{tabular}{cccc}
\hline Entry & Base (3 equiv.) & Solvent $(15 \mathrm{ml})$ & $\%$ Yield \\
\hline 1 & $\mathrm{KOH}$ & Water & 46 \\
2 & $\mathrm{KOH}$ & Ethanol & 51 \\
3 & ---- & Ethanol & Trace \\
4 & $\mathrm{NaOH}$ & Water & 40 \\
$\mathbf{5}$ & Piperidine & Ethanol & $\mathbf{8 8}$ \\
6 & Triethylamine & Ethanol & 78 \\
7 & DBU & Ethanol & 40 \\
8 & $N-$ Methylpiperidine & Ethanol & 78 \\
9 & Piperidine & Benzene & 60 \\
10 & Pyridine & Ethanol & 20 \\
11 & Pyridine & ---- & 30 \\
12 & Piperidine & ---- & 50 \\
\hline
\end{tabular}

The workup procedure is simple. The crude reaction mixture was allowed to cool at room temperature. In some reactions, the crude product precipitated from solution and was collected by filtration and washed several times with dichloromethane/hexane mixture $(60: 40, \mathrm{v} / \mathrm{v})$ and give the pure product after recrystallization from ethanol. In those reactions in which the product did not precipitate from solution, the excess solvent (ethanol) was removed in vacuum and the solid product obtained was triturated with dichloromethane/hexane mixture and give the pure product after recrystallization from ethanol.

Further evaluation of the data in Table 2 reveal that condensation of symmetrically substituted 1,2-phenylenediamine 3a-c with a variety of indole based aldehydes, prepared from the corresponding acid chloride $\mathbf{2 a - f}$ in presence of $\mathrm{HSnBu}_{3}$, furnishes (1H-indol-3-yl)quinoxalines $\mathbf{4 a}$, $\mathbf{4 h}, \mathbf{4 i}$, and $\mathbf{4 k}$, and 6,7-disubstituted $1 H$-indol-3-yl)quinoxalines $\mathbf{4 b - g}, \mathbf{4 j}, \mathbf{4 l}$ and $\mathbf{4 m}$ respectively. In addition, 1,2-phenylenediamines substituted with the strong electron-withdrawing group (EWG) carbethoxy, nitro, or cyano at the para position 3d-f, provides the corresponding 6- substituted-2(1H-indol-3-yl)quinoxalines 4n-p. Direct electronic delocalization occurs between the 4-EWG substituent and 1-amino group. This decreases the basicity and hence nucleophilicity of the 1amine, thus it is the 2-amino group that is the active nucleophile in these reactions.

However, the difference in nucleophilicity of the two amino groups in 1,2-phenylenediamine substituted with the weakly electron-donating methyl group $\mathbf{3 g}$ is not so large which results in the production of an inseparable mixture of the 6-methyl- 6 and 7-methyl isomers 5 of (1H-indol-3yl)quinoxaline (see Scheme 2). The ${ }^{1} \mathrm{H}$ NMR shows two different peaks at $\delta 11.53 \mathrm{ppm}, \delta 11.51$ ppm for two $\mathrm{NH}$ protons and at $\delta 9.16 \mathrm{ppm}, \delta 9.13 \mathrm{ppm}$ for two $\mathrm{N}=\mathrm{CH}$ protons. 
Table 2. Synthesis of indole based quinoxalines 4a-p

$$
\text { Quinoxalines 4a-p }{ }^{\mathrm{a}}
$$

\begin{tabular}{|c|c|c|c|c|}
\hline Entry & $\begin{array}{c}\text { Acid chlorides } \\
\mathbf{2 a - f}\end{array}$ & $\begin{array}{l}\text { 1,2-Diamines } \\
\text { 3a-f }\end{array}$ & & $\%$ Yield $^{\mathrm{b}}$ \\
\hline 1 & $\begin{array}{c}\mathrm{R}_{1}=\mathrm{R}_{2}=\mathrm{H} \\
\mathbf{2 a}\end{array}$ & $\begin{array}{c}\mathrm{R}_{3}= \\
\quad \mathrm{R}_{4}=\mathrm{H}\end{array}$ & $\begin{array}{c}\mathrm{R}_{1}=\mathrm{R}_{2}=\mathrm{R}_{3}=\mathrm{R}_{4}=\mathrm{H} \\
\mathbf{4 a}\end{array}$ & 88 \\
\hline 2 & $\begin{array}{c}\mathrm{R}_{1}=\mathrm{R}_{2}=\mathrm{H} \\
\mathbf{2 a}\end{array}$ & $\begin{array}{c}\mathrm{R}_{3}=\mathrm{R}_{4}=\mathrm{CH}_{3} \\
\mathbf{3 b}\end{array}$ & $\begin{array}{c}\mathrm{R}_{1}=\mathrm{R}_{2}=\mathrm{H} ; \mathrm{R}_{3}=\mathrm{R}_{4}=\mathrm{CH}_{3} \\
\mathbf{4 b}\end{array}$ & 93 \\
\hline 3 & $\begin{array}{c}\mathrm{R}_{1}=\mathrm{R}_{2}=\mathrm{H} \\
\mathbf{2 a}\end{array}$ & $\begin{array}{c}\mathrm{R}_{3}=\mathrm{R}_{4}=\mathrm{Cl} \\
\text { 3c }\end{array}$ & $\begin{array}{c}\mathrm{R}_{1}=\mathrm{R}_{2}=\mathrm{H} ; \mathrm{R}_{3}=\mathrm{R}_{4}=\mathrm{Cl} \\
\mathbf{c}\end{array}$ & 80 \\
\hline 4 & $\begin{array}{c}\mathrm{R}_{1}=\mathrm{OCH}_{3}, \mathrm{R}_{2}=\mathrm{H} \\
\mathbf{2 b}\end{array}$ & $\begin{array}{c}\mathrm{R}_{3}=\mathrm{R}_{4}=\mathrm{CH}_{3} \\
\mathbf{3 b}\end{array}$ & $\mathrm{R}_{1}=\mathrm{OCH}_{3}, \mathrm{R}_{2}=\mathrm{H} ; \mathrm{R}_{3}=\mathrm{R}_{4}=\mathrm{CH}_{3}$ & 95 \\
\hline 5 & $\begin{array}{c}\mathrm{R}_{1}=\mathrm{Cl}, \mathrm{R}_{2}=\mathrm{H} \\
\mathbf{2 c}\end{array}$ & $\begin{array}{c}\mathrm{R}_{3}=\mathrm{R}_{4}=\mathrm{CH}_{3} \\
\mathbf{3 b}\end{array}$ & $\begin{array}{c}\mathrm{R}_{1}=\mathrm{Cl}, \mathrm{R}_{2}=\mathrm{H} ; \mathrm{R}_{3}=\mathrm{R}_{4}=\mathrm{CH}_{3} \\
\mathbf{e}\end{array}$ & 90 \\
\hline 6 & $\begin{array}{c}\mathrm{R}_{1}=\mathrm{CN}, \mathrm{R}_{2}=\mathrm{H} \\
\mathbf{2 d}\end{array}$ & $\begin{array}{c}\mathrm{R}_{3}=\mathrm{R}_{4}=\mathrm{CH}_{3} \\
\mathbf{3 b}\end{array}$ & $\mathrm{R}_{1}=\mathrm{CN}, \mathrm{R}_{2}=\underset{\mathbf{4 f}}{\mathrm{H} ; \mathrm{R}_{3}}=\mathrm{R}_{4}=\mathrm{CH}_{3}$ & 91 \\
\hline 7 & $\begin{array}{c}\mathrm{R}_{1}=\mathrm{NO}_{2}, \mathrm{R}_{2}=\mathrm{H} \\
\mathbf{2} \mathbf{e}\end{array}$ & $\begin{array}{c}\mathrm{R}_{3}=\underset{\mathbf{3 b}}{\mathrm{R}_{4}}=\mathrm{CH}_{3} \\
\end{array}$ & 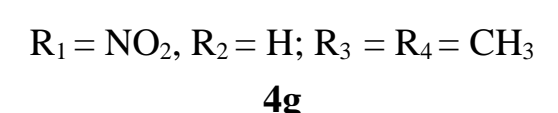 & 85 \\
\hline 8 & $\begin{array}{c}\mathrm{R}_{1}=\mathrm{Cl}, \mathrm{R}_{2}=\mathrm{H} \\
\mathbf{2 c}\end{array}$ & $\begin{array}{c}\mathrm{R}_{3}=\mathrm{R}_{4}=\mathrm{H} \\
\mathbf{3 a}\end{array}$ & $\begin{array}{c}\mathrm{R}_{1}=\mathrm{Cl} ; \mathrm{R}_{2}=\mathrm{R}_{3}=\mathrm{R}_{4}=\mathrm{H} \\
\mathbf{4 h}\end{array}$ & 89 \\
\hline 9 & $\begin{array}{c}\mathrm{R}_{1}=\mathrm{CN}, \mathrm{R}_{2}=\mathrm{H} \\
\mathbf{2 d}\end{array}$ & $\begin{array}{c}\mathrm{R}_{3}= \\
\mathbf{3 a}\end{array}$ & 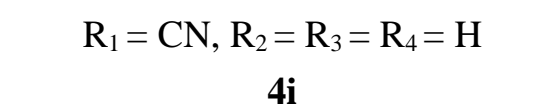 & 85 \\
\hline 10 & $\begin{array}{c}\mathrm{R}_{1}=\mathrm{CN}, \mathrm{R}_{2}=\mathrm{H} \\
\mathbf{2 d}\end{array}$ & $\begin{array}{c}\mathrm{R}_{3}=\mathrm{R}_{4}=\mathrm{Cl} \\
\mathbf{3 c}\end{array}$ & $\mathrm{R}_{1}=\mathrm{CN}, \mathrm{R}_{2}=\underset{\mathbf{4 j}}{\mathrm{H}} ; \mathrm{R}_{3}=\mathrm{R}_{4}=\mathrm{Cl}$ & 90 \\
\hline 11 & $\begin{array}{c}\mathrm{R}_{1}=\underset{\mathbf{2 f}}{\mathrm{OCH}_{3}, \mathrm{R}_{2}}=\mathrm{CH}_{3} \\
\end{array}$ & $\begin{array}{c}\mathrm{R}_{3}= \\
\quad \mathrm{R}_{4}=\mathrm{H}\end{array}$ & $\begin{array}{c}\mathrm{R}_{1}=\mathrm{OCH}_{3}, \mathrm{R}_{2}=\mathrm{CH}_{3} \\
\mathrm{R}_{3}=\underset{\mathbf{R}}{\mathbf{4}}=\mathrm{H}\end{array}$ & 88 \\
\hline 12 & $\begin{array}{c}\mathrm{R}_{1}=\mathrm{OCH}_{3}, \mathrm{R}_{2}=\mathrm{CH}_{3} \\
\mathbf{2 f}\end{array}$ & $\begin{array}{c}\mathrm{R}_{3}=\mathrm{R}_{4}=\mathrm{CH}_{3} \\
\mathbf{3 b}\end{array}$ & $\mathrm{R}_{1}=\mathrm{OCH}_{3}, \mathrm{R}_{2}=\mathrm{R}_{3}=\mathrm{R}_{4}=\mathrm{CH}_{3}$ & 90 \\
\hline 13 & $\begin{array}{c}\mathrm{R}_{1}=\mathrm{OCH}_{3}, \mathrm{R}_{2}=\mathrm{CH}_{3} \\
\mathbf{2 f}\end{array}$ & $\begin{array}{c}\mathrm{R}_{3}=\mathrm{R}_{4}=\mathrm{Cl} \\
\mathbf{3 c}\end{array}$ & $\mathrm{R}_{1}=\mathrm{OCH}_{3} ; \mathrm{R}_{2}=\underset{\mathbf{4 m}}{\mathrm{CH}_{3} \mathrm{R}_{3}}=\mathrm{R}_{4}=\mathrm{Cl}$ & 88 \\
\hline 14 & $\underset{\mathbf{2 f}}{\mathrm{R}_{1}=\mathrm{OCH}_{3}, \mathrm{R}_{2}=\mathrm{CH}_{3}}$ & $\begin{array}{c}\mathrm{R}_{3}=\mathrm{H}, \mathrm{R}_{4}= \\
\mathrm{COOCH}_{3} \\
\mathbf{3 d}\end{array}$ & $\begin{array}{c}\mathrm{R}_{1}=\mathrm{OCH}_{3}, \mathrm{R}_{2}=\mathrm{CH}_{3} ; \mathrm{R}_{3}=\mathrm{H}, \mathrm{R}_{4}= \\
\mathrm{COOCH}_{3} \\
\mathbf{4 n}\end{array}$ & 91 \\
\hline
\end{tabular}


Table 2. Continued

\begin{tabular}{|c|c|c|c|c|}
\hline \multirow[b]{2}{*}{ Entry } & Acid chlorides & 1,2-Diamines & Quinoxalines $\mathbf{4 a -} \mathbf{p}^{\mathrm{a}}$ & \multirow[b]{2}{*}{$\%$ Yield $^{b}$} \\
\hline & & & & \\
\hline & $\mathrm{R}_{1}=\mathrm{OCH}_{3}, \mathrm{R}_{2}=\mathrm{CH}_{3}$ & $\mathrm{R}_{3}=\mathrm{H}, \mathrm{R}_{4}=\mathrm{NO}_{2}$ & $\mathrm{R}_{1}=\mathrm{OCH}_{3}, \mathrm{R}_{2}=\mathrm{CH}_{3}$ & \\
\hline 15 & $2 f$ & $3 e$ & $\begin{array}{c}\mathrm{R}_{3}=\mathrm{H}, \mathrm{R}_{4}=\mathrm{NO}_{2} \\
\mathbf{4 o}\end{array}$ & 89 \\
\hline 16 & $\begin{array}{c}\mathrm{R}_{1}=\mathrm{R}_{2}=\mathrm{H} \\
\mathbf{2 a}\end{array}$ & $\begin{array}{c}\mathrm{R}_{3}=\mathrm{H}, \mathrm{R}_{4}=\mathrm{CN} \\
\mathbf{3 f}\end{array}$ & $\begin{array}{c}\mathrm{R}_{1}=\mathrm{R}_{2}=\mathrm{H} ; \mathrm{R}_{3}=\mathrm{H}, \mathrm{R}_{4}=\mathrm{CN} \\
\mathbf{4 p}\end{array}$ & 78 \\
\hline
\end{tabular}

${ }^{\mathrm{a}}$ All the compounds were characterized by ${ }^{1} \mathrm{H}$ NMR, ${ }^{13} \mathrm{C}$ NMR, IR and HRMS analysis. ${ }^{\mathrm{b}}$ Isolated yield.<smiles>O=CC(=O)c1c[nH]c2ccccc12</smiles><smiles>Cc1ccc(N)c(N)c1</smiles>

$3 g$<smiles>C/C=C\C=C/C=C\c1c[nH]c2ccccc12</smiles>

5<smiles>Cc1ccc2nc(-c3c[nH]c4ccccc34)cnc2c1</smiles>

6

Scheme 2. Reaction of 2-(1H-indol-3-yl)-2-oxoacetaldehyde with 4-methyl-1,2-phenylenediamine.

Finally, a possible mechanism for the synthesis of quinoxaline is shown in Scheme 3.<smiles>CCCC(=O)C(=O)c1c[nH]c2ccccc12</smiles><smiles>NC(=O)c1c[nH]c2ccc(O)cc12</smiles>

Schiff Base

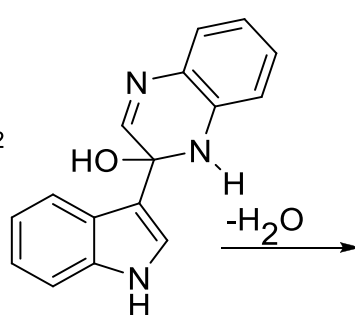

$\mathrm{H}$<smiles>c1ccc2nc(-c3c[nH]c4ccccc34)cnc2c1</smiles>

Scheme 3. Mechanism for the formation of quinoxaline. 
As shown, the aldehydes and diamines react to give a Schiff base that undergoes successive intramolecular cyclisation and dehydration to give $\mathbf{4 a}$.

\section{Conclusions}

In summary we have successfully developed an easy access to novel series of indole based biologically important quinoxalines. This method is more efficient than previously reported. ${ }^{12} \mathrm{We}$ are currently investigating the synthesis of a number of other quinoxaline-based drug molecules by this method and work is in progress for the detail biological activity (antibacterial, antifungal, anticancer and neuroprotective kinase inhibitor activity) of these important compounds. Results in these areas will be presented in due course.

\section{Experimental Section}

General. The ${ }^{1} \mathrm{H}$ and ${ }^{13} \mathrm{C}$ NMR spectra were recorded on $500 \mathrm{MHz}$ Jeol multinuclear NMR spectrometer; chemical shifts were referenced to tetramethylsilane (TMS) as internal standard. Infrared (IR) spectra were obtained on a Varian 3100 Fourier transform (FT) IR Spectrometer. Melting points were taken on a Meltemp apparatus. All chemicals and reagents were purchased from commercial sources. Mass spectra was obtained from Washington University, St. Louis.

\section{General procedure for the synthesis of acid chlorides (2a-f)}

To a solution of appropriate indole (1 equiv) in anhydrous diethyl ether $(120 \mathrm{~mL})$ at $0{ }^{\circ} \mathrm{C}$, oxalyl chloride (1.3 equiv.) was added drop wise over $30 \mathrm{~min}$. The reaction mixture was stirred at $0{ }^{\circ} \mathrm{C}$ for $3 \mathrm{~h}$, then allowed to warm at room temperature and stirred for $1 \mathrm{~h}$. The resulting solid products were collected by filtration, washed with cold anhydrous diethyl ether $(100 \mathrm{~mL})$ and dried under vacuum to yield 2a-f. All the compounds were well characterized with ${ }^{1} \mathrm{H}$ NMR, ${ }^{13} \mathrm{C}$ NMR and IR.

2-(1H-Indol-3-yl)-2-oxoacetyl chloride (2a). Obtained as yellow crystals. Yield $=90 \%$. Decomposition point: $117-119^{\circ} \mathrm{C}$. All the chemical and physical data are identical to previously reported. $^{10}$

2-(5-Methoxy-1H-indol-3-yl)-2-oxoacetyl chloride (2b). Obtained as bright orange solid. Decomposition point: $238-239{ }^{\circ} \mathrm{C}$. Yield $=80 \%$. IR $\left(\mathrm{KBr}, \mathrm{cm}^{-1}\right): 3194,1778,1617$ which are in accordance with those previously reported. ${ }^{11}{ }^{1} \mathrm{H}$ NMR (DMSO- $\left.d_{6}\right) \delta 12.31$ (brs, $\left.1 \mathrm{H}, \mathrm{NH}\right), 8.29(\mathrm{~d}, J$ $=5.7 \mathrm{~Hz}, 1 \mathrm{H}, \mathrm{Ar}-\mathrm{CH}), 7.63(\mathrm{~d}, J=5.7 \mathrm{~Hz}, 1 \mathrm{H}, \mathrm{Ar}-\mathrm{CH}), 7.41(\mathrm{~d}, J=8.5 \mathrm{~Hz}, 1 \mathrm{H}, \mathrm{Ar}-\mathrm{CH}), 6.87(\mathrm{dd}$, $J=5.7 \mathrm{~Hz}, 8.5 \mathrm{~Hz}, 1 \mathrm{H}, \mathrm{Ar}-\mathrm{CH}), 3.75\left(\mathrm{~s}, 3 \mathrm{H}, \mathrm{OCH}_{3}\right) .{ }^{13} \mathrm{C} \mathrm{NMR}\left(\mathrm{DMSO}-d_{6}\right) \delta 181.1(\mathrm{C}=\mathrm{O}), 165.8$ $(\mathrm{C}=\mathrm{O}), 156.5(\mathrm{C}), 138.3(\mathrm{CH}), 131.9(\mathrm{C}), 127.0(\mathrm{C}), 114.0(\mathrm{CH}), 113.7(\mathrm{C}), 112.6(\mathrm{C}), 103.5(\mathrm{C})$, $55.8\left(\mathrm{OCH}_{3}\right)$.

2-(5-Chloro-1H-indol-3-yl)-2-oxoacetyl chloride (2c). Obtained as yellow powder. Yield $=88 \%$. Decomposition point: $157-158{ }^{\circ} \mathrm{C}$. All the chemical and physical data were identical to those previously reported. ${ }^{10}$ 
2-(5-Cyano-1H-indol-3-yl)-2-oxoacetyl chloride (2d). Obtained as brick red solid. Yield $=93 \%$. Decomposition point: $184-185{ }^{\circ} \mathrm{C}$. IR $\left(\mathrm{KBr}, \mathrm{cm}^{-1}\right): 3202,2220,1733,1648 .{ }^{1} \mathrm{H}$ NMR (DMSO- $\left.d_{6}\right) \delta$ 12.02 (brs, $1 \mathrm{H}, \mathrm{NH}), 8.60$ (d, $J=2.8 \mathrm{~Hz}, 1 \mathrm{H}, \mathrm{Ar}-\mathrm{CH}), 8.48$ (s, $1 \mathrm{H}, \mathrm{Ar}-\mathrm{CH}), 7.70(\mathrm{~d}, J=8.6 \mathrm{~Hz}, 1 \mathrm{H}$, Ar-CH), $7.63(\mathrm{dd}, J=2.8 \mathrm{~Hz}, 8.6 \mathrm{~Hz}, 1 \mathrm{H}, \mathrm{Ar}-\mathrm{CH}) .{ }^{13} \mathrm{C}$ NMR (DMSO- $\left.d_{6}\right) \delta 181.1(\mathrm{C}=\mathrm{O}), 164.9$ $(\mathrm{C}=\mathrm{O}), 140.6(\mathrm{C}), 139.0(\mathrm{C}), 127.1(\mathrm{CH}), 126.4(\mathrm{CH}), 126.0(\mathrm{CH}), 120.1(\mathrm{CH}), 114.7(\mathrm{CN}), 112.9$ (C), $105.3(\mathrm{C})$.

2-(5-Nitro-1H-indol-3-yl)-2-oxoacetyl chloride (2e). Obtained as pale yellow solid. Yield $=91 \%$. Decomposition point: $250-252{ }^{\circ} \mathrm{C}$. IR $\left(\mathrm{KBr}, \mathrm{cm}^{-1}\right): 3201,1743,1647,1508 .{ }^{1} \mathrm{H}$ NMR (DMSO- $\left.d_{6}\right) \delta$ 12.09 (brs, $1 \mathrm{H}, \mathrm{NH}), 8.94(\mathrm{~d}, J=2.3 \mathrm{~Hz}, 1 \mathrm{H}, \mathrm{Ar}-\mathrm{CH}), 8.65(\mathrm{~d}, J=2.3 \mathrm{~Hz}, 1 \mathrm{H}, \mathrm{Ar}-\mathrm{CH}), 8.10$ (dd, $J$ $=2.3 \mathrm{~Hz}, 8.5 \mathrm{~Hz}, 1 \mathrm{H}, \mathrm{Ar}-\mathrm{CH}), 7.69(\mathrm{~d}, J=8.5 \mathrm{~Hz}, 1 \mathrm{H}, \mathrm{Ar}-\mathrm{CH}) .{ }^{13} \mathrm{C}$ NMR (DMSO- $\left.d_{6}\right) \delta 181.2$ $(\mathrm{C}=\mathrm{O}), 164.8(\mathrm{C}=\mathrm{O}), 143.7(\mathrm{C}), 141.7(\mathrm{CH}), 140.3(\mathrm{C}), 125.6(\mathrm{C}), 119.5(\mathrm{CH}), 117.8(\mathrm{CH}), 114.0$ $(\mathrm{CH})$.

2-(5-Methoxy-2-methyl-1H-indol-3-yl)-2-oxoacetyl chloride (2f). Obtained as dark red solid. Decomposition point: $131-133{ }^{\circ} \mathrm{C}$. Yield $=88 \%$. IR $\left(\mathrm{KBr}, \mathrm{cm}^{-1}\right): 3200,1797,1738,1575 .{ }^{1} \mathrm{H}$ NMR $\left(\mathrm{DMSO}-d_{6}\right) \delta 12.32$ (brs, $\left.1 \mathrm{H}, \mathrm{NH}\right), 7.45(\mathrm{~d}, J=2.8 \mathrm{~Hz}, 1 \mathrm{H}, \mathrm{Ar}-\mathrm{CH}), 7.29$ (d, $J=9.1 \mathrm{~Hz}, 1 \mathrm{H}, \mathrm{Ar}-$ $\mathrm{CH}), 6.78(\mathrm{dd}, J=2.8 \mathrm{~Hz}, 9.1 \mathrm{~Hz}, 1 \mathrm{H}, \mathrm{Ar}-\mathrm{CH}) .{ }^{13} \mathrm{C}$ NMR (DMSO-d $\left.)\right) \delta 183.6(\mathrm{C}=\mathrm{O}), 168.7(\mathrm{C}=\mathrm{O})$, $156.1(\mathrm{C}), 147.6(\mathrm{C}), 130.2(\mathrm{C}), 127.9(\mathrm{C}), 112.8(\mathrm{CH}), 112.5(\mathrm{CH}), 108.6(\mathrm{C}), 103.1(\mathrm{CH}), 55.7$ $\left(\mathrm{OCH}_{3}\right), 13.8\left(\mathrm{CH}_{3}\right)$.

General procedure for the synthesis of suitably substituted 2-(1H-indol-3-yl)-2oxoacetaldehyde

To a suspension of oxoacetyl chloride $(25 \mathrm{mmol})$ in ethyl acetate $(80 \mathrm{~mL})$ at $0{ }^{\circ} \mathrm{C}$ was added a solution of tributyltin hydride $(25 \mathrm{mmol})$. The reaction mixture was stirred at $0{ }^{\circ} \mathrm{C}$ for $30 \mathrm{~min}$, warmed to room temperature and then stirred for an additional $15 \mathrm{~h}$. Hexane $(100 \mathrm{~mL})$ was added and the resulting solid was collected by filtration, washed with copious amounts of hexane, then dried under vacuum to give ketoaldehyde (60\% yield) which was immediately subjected to the next step without further purification.

General procedure for the synthesis of quinoxalines using preparation of (4a) as typical example

To a solution of keto aldehyde obtained from $2 \mathbf{a}(0.4 \mathrm{~g}, 2.31 \mathrm{mmol})$ and 1,2-phenylenediamine $(0.27 \mathrm{~g}, 2.31 \mathrm{mmol})$ in $15 \mathrm{~mL}$ of ethanol at $90{ }^{\circ} \mathrm{C}$ was added piperidine $(0.98 \mathrm{~g}, 11.5 \mathrm{mmol})$. After stirring at $90{ }^{\circ} \mathrm{C}$ for $3 \mathrm{hr}$, the reaction mixture was allowed to cool at room temperature. The solid formed was collected by filtration, washed with cold ethanol $(50 \mathrm{~mL})$, dichloromethane/hexane mixture $(50 \mathrm{~mL}, 60: 40, \mathrm{v} / \mathrm{v})$ to afford the desired product $4 \mathbf{a}$ which was recrystalized from ethanol.

2-(1H-Indol-3-yl)quinoxaline (4a). This compound was obtained as yellow powder. Mp 203-204 ${ }^{\circ} \mathrm{C}$ (lit ${ }^{12}$ m.p. $\left.202-203{ }^{\circ} \mathrm{C}\right)$.

2-(1H-Indol-3-yl)-6,7-dimethylquinoxaline (4b). This compound was obtained as light yellow crystalline solid. Mp 279-281 ${ }^{\circ} \mathrm{C}$. IR $\left(\mathrm{KBr}, \mathrm{cm}^{-1}\right): 3432(\mathrm{NH}) .{ }^{1} \mathrm{H}$ NMR (DMSO- $\left.d_{6}\right): \delta 12.21$ (brs, $1 \mathrm{H}, \mathrm{NH}), 9.33$ (s, 1H, Ar-CH)), 8.74 (dd, $J=3.1 \mathrm{~Hz}, 7.8 \mathrm{~Hz}, 1 \mathrm{H}, \mathrm{Ar}-\mathrm{CH}), 8.50$ (s, 1H, Ar-CH), $7.81(\mathrm{~s}, 1 \mathrm{H}, \mathrm{Ar}-\mathrm{CH}), 7.71$ (s, 1H, Ar-CH), 7.20-7.19 (m, 1H, Ar-CH), 7.19-7.18 (m, 2H, Ar-CH), 
$2.42\left(\mathrm{~s}, 3 \mathrm{H}, \mathrm{CH}_{3}\right), 2.39\left(\mathrm{~s}, 3 \mathrm{H}, \mathrm{CH}_{3}\right) .{ }^{13} \mathrm{C}$ NMR (DMSO- $\left.d_{6}\right) \delta 150.6(\mathrm{C}), 143.9(\mathrm{CH}), 141.1(\mathrm{C})$, $140.4(\mathrm{C}), 138.7(\mathrm{C}), 138.0(\mathrm{C}), 137.8(\mathrm{C}), 129.09(\mathrm{CH}), 128.3(\mathrm{CH}), 128.1(\mathrm{CH}), 122.9(\mathrm{CH})$, 122.8 (CH), $121.1(\mathrm{CH}), 111.2(\mathrm{CH}), 20.3\left(\mathrm{CH}_{3}\right), 20.1\left(\mathrm{CH}_{3}\right)$. HRMS: Calcd $[\mathrm{M}+\mathrm{H}]^{+}$for $\mathrm{C}_{18} \mathrm{H}_{15} \mathrm{~N}_{3}$ : 274.1339. Found: 274.1347.

6,7-Dichloro-2-(1H-indol-3-yl)quinoxaline (4c). This compound was obtained as bottle green solid. Mp 220-222 ${ }^{\circ} \mathrm{C}$. IR $\left(\mathrm{KBr}, \mathrm{cm}^{-1}\right): 3335(\mathrm{NH}) .{ }^{1} \mathrm{H}$ NMR (DMSO-d $\left.d_{6}\right) \delta 12.31$ (brs, $1 \mathrm{H}, \mathrm{NH}$ ), 9.49 (s, $1 \mathrm{H}, \mathrm{Ar}-\mathrm{CH}), 8.68(\mathrm{~d}, J=8.5 \mathrm{~Hz}, 1 \mathrm{H}, \mathrm{Ar}-\mathrm{CH}), 8.30(\mathrm{~s}, 2 \mathrm{H}, \mathrm{Ar}-\mathrm{CH}), 7.50-7.22$ (m, 4H, Ar$\mathrm{CH}) .{ }^{13} \mathrm{C}$ NMR (DMSO- $\left.d_{6}\right) \delta 150.6(\mathrm{C}), 143.9(\mathrm{C}), 141.18(\mathrm{C}), 140.4(\mathrm{C}), 138.0(\mathrm{C}), 137.8(\mathrm{C})$, $128.3(\mathrm{CH}), 128.1(\mathrm{CH}), 122.9(\mathrm{CH}), 122.8(\mathrm{CH}), 121.1(\mathrm{CH}), 111.2(\mathrm{CH})$. HRMS: Calcd $[\mathrm{M}+\mathrm{H}]^{+}$ for $\mathrm{C}_{16} \mathrm{H}_{9} \mathrm{~N}_{3} \mathrm{Cl}_{2}: 314.0255$. Found: 314.0248 .

2-(5-Methoxy-1H-indol-3-yl)-6,7-dimethylquinoxaline (4d). This compound was obtained as bright yellow powder. Mp 277-280 ${ }^{\circ} \mathrm{C}$. IR $\left(\mathrm{KBr}, \mathrm{cm}^{-1}\right)$ : $3431(\mathrm{NH}) .{ }^{1} \mathrm{H}$ NMR (DMSO- $\left.d_{6}\right) \delta 12.01$ (brs, 1H, NH), 9.30 (s, 1H, Ar-CH), 8.45 (s, 1H, Ar-CH), 8.29 (d, J = 2.5 Hz, 1H, Ar-CH), 7.79 (s, 1H, Ar-CH), 7.70 (s, 1H, Ar-CH), 7.37 (s, 1H, Ar-CH), 6.85-6.83 (m, 1H, Ar-CH), 2.99 (s, 3H, $\mathrm{OCH}_{3}$ ), 2.42 (s, 3H, CH 3 ), 2.39 (s, 3H, $\mathrm{CH}_{3}$ ). ${ }^{13} \mathrm{C}$ NMR (DMSO-d $) \delta 155.07$ (C), $150.84(\mathrm{C}$ ), $143.90(\mathrm{CH}), 141.16(\mathrm{C}), 140.31(\mathrm{C}), 138.63(\mathrm{C}), 137.8(\mathrm{C}), 133.13(\mathrm{C}), 129.79(\mathrm{C}), 128.37(\mathrm{CH})$, $128.13(\mathrm{CH}), 112.57(\mathrm{CH}), 104.81(\mathrm{CH}), 55.90\left(\mathrm{OCH}_{3}\right), 20.30,\left(\mathrm{CH}_{3}\right), 20.15\left(\mathrm{CH}_{3}\right)$. HRMS: Calcd $[\mathrm{M}+\mathrm{H}]^{+}$for $\mathrm{C}_{19} \mathrm{H}_{17} \mathrm{~N}_{3} \mathrm{O}: 304.1444$. Found: 304.1457 .

2-(5-Chloro-1H-indol-3-yl)-6,7-dimethylquinoxaline (4e). This compound was obtained as brownish yellow powder. Mp 296-297 ${ }^{\circ} \mathrm{C}$. IR $\left(\mathrm{KBr}, \mathrm{cm}^{-1}\right)$ : $3337(\mathrm{NH}) .{ }^{1} \mathrm{H}$ NMR (DMSO- $\left.d_{6}\right) \delta$ 11.98 (brs, 1H, NH), 9.31 (s, 1H, Ar-CH), 8.73 (d, $J=2.3 \mathrm{~Hz}, 1 \mathrm{H}, \mathrm{Ar}-\mathrm{CH}), 8.58$ (s, 1H, Ar-CH), 7.81 (s, $1 \mathrm{H}, \mathrm{Ar}-\mathrm{CH}), 7.70$ (s, $1 \mathrm{H}, \mathrm{Ar}-\mathrm{CH}), 7.54-7.52(\mathrm{~m}, 1 \mathrm{H}, \mathrm{Ar}-\mathrm{CH}), 7.20$ (dd, J = 2.5 Hz, 7.8 Hz, $1 \mathrm{H}, \mathrm{Ar}-\mathrm{CH}$ ), $2.42\left(\mathrm{~s}, 3 \mathrm{H}, \mathrm{CH}_{3}\right) .2 .39\left(\mathrm{~s}, 3 \mathrm{H}, \mathrm{CH}_{3}\right) .{ }^{13} \mathrm{C}$ NMR (DMSO-d 6$) \delta 150.20(\mathrm{C}), 143.80$ $(\mathrm{CH}), 141.04(\mathrm{C}), 140.58(\mathrm{C}), 138.8(\mathrm{C}), 138.2(\mathrm{C}), 136.7(\mathrm{C}), 131.05(\mathrm{CH}), 128.37(\mathrm{CH}), 128.12$ (C), $125.6(\mathrm{C}), 122.7(\mathrm{CH}), 121.7(\mathrm{CH}), 114.4 \mathrm{CH}), 113.0(\mathrm{C}), 20.23\left(\mathrm{CH}_{3}\right), 20.15\left(\mathrm{CH}_{3}\right)$. HRMS: Calcd $[\mathrm{M}+\mathrm{H}]^{+}$for $\mathrm{C}_{18} \mathrm{H}_{14} \mathrm{~N}_{3} \mathrm{Cl}$ : 308.0949. Found: 308.0963 .

3-(6,7-Dimethylquinoxalin-2-yl)- $\mathbf{H}$-indole-5-carbonitrile (4f). This compound was obtained as brownish solid. Mp 275-277 ${ }^{\circ} \mathrm{C}$. IR (KBr, cm ${ }^{-1}$ ): $3438(\mathrm{NH}), 2221(\mathrm{CN}) .{ }^{1} \mathrm{H}$ NMR (DMSO- $\left.d_{6}\right) \delta$ 12.32 (brs, 1H, NH), 9.16 (s, 1H, Ar-CH), 8.97 (s, 1H, Ar-CH), 7.65-7.54 (m, 4H, Ar-CH), 7.00 (s, $1 \mathrm{H}, \mathrm{Ar}-\mathrm{CH}$ ), 2.27 (s, $\left.3 \mathrm{H}, \mathrm{CH}_{3}\right), 2.24\left(\mathrm{~s}, 3 \mathrm{H}, \mathrm{CH}_{3}\right) .{ }^{13} \mathrm{C}$ NMR (DMSO-d $\left.d_{6}\right) \delta 154.8(\mathrm{C}), 150.6(\mathrm{C})$, $138.5(\mathrm{C}), 134.8(\mathrm{CH}), 132.4(\mathrm{C}), 131.5(\mathrm{C}), 128.4(\mathrm{CH}), 128.3(\mathrm{CH}), 126.3(\mathrm{C}), 125.8(\mathrm{CH})$, $121.24(\mathrm{C}), 115.5(\mathrm{CH}), 113.7(\mathrm{CH}), 112.3(\mathrm{C}), 103.3(\mathrm{C}), 20.2\left(\mathrm{CH}_{3}\right), 19.4\left(\mathrm{CH}_{3}\right)$. HRMS: Calcd $[\mathrm{M}+\mathrm{H}]^{+}$for $\mathrm{C}_{19} \mathrm{H}_{14} \mathrm{~N}_{4}: 299.1298$. Found: 299.1311 .

6,7-Dimethyl-2-(5-nitro-1H-indol-3-yl)quinoxaline (4g). This compound was obtained as light yellow solid. Mp > $300{ }^{\circ} \mathrm{C}$. IR $\left(\mathrm{KBr}, \mathrm{cm}^{-1}\right): 3370(\mathrm{NH}) .{ }^{1} \mathrm{H}$ NMR (DMSO- $\left.d_{6}\right) \delta 12.41$ (brs, $1 \mathrm{H}, \mathrm{NH}$ ), $9.58(\mathrm{~s}, 1 \mathrm{H}, \mathrm{Ar}-\mathrm{CH}), 8.70(\mathrm{~s}, 1 \mathrm{H}, \mathrm{Ar}-\mathrm{CH}), 8.57$ (s, 1H, Ar-CH), 8.07 (s, 2H, Ar-CH), 7.76-7.62 (m, $2 \mathrm{H}, \mathrm{Ar}-\mathrm{CH}$ ), 2.47 (s, 3H, $\mathrm{CH}_{3}$ ), 2.43 (s, 3H, $\mathrm{CH}_{3}$ ). ${ }^{13} \mathrm{C} \mathrm{NMR}$ (DMSO-d 6 ): $\delta 149.2(\mathrm{C}), 143.6(\mathrm{CH})$, $140.8(\mathrm{C}), 140.6(\mathrm{C}), 139.1(\mathrm{C}), 138.9(\mathrm{C}), 137.3(\mathrm{CH}), 131.9(\mathrm{CH}), 128.4(\mathrm{CH}), 119.6(\mathrm{CH}), 118.7$ $(\mathrm{CH}), 118.3(\mathrm{CH}), 115.4(\mathrm{CH}), 113.0(\mathrm{CH}), 20.16\left(\mathrm{CH}_{3}\right)$. HRMS: Calcd $[\mathrm{M}+\mathrm{H}]^{+}$for $\mathrm{C}_{18} \mathrm{H}_{14} \mathrm{~N}_{4} \mathrm{O}_{2}$ : 319.1190. Found: 319.1192. 
2-(5-Chloro-1H-indol-3-yl)quinoxaline (4h). This compound was obtained as dark brown solid. Mp 225-227 ${ }^{\circ} \mathrm{C}$. IR (KBr, cm ${ }^{-1}$ ): $3330(\mathrm{NH}) .{ }^{1} \mathrm{H}$ NMR (DMSO-d $) \delta 12.19$ (brs, 1H, NH), 9.47 (s, $1 \mathrm{H}, \mathrm{ArCH}), 8.75$ (s, 1H, Ar-CH), 8.67 (s, 1H, Ar-CH), 7.97 (dd, J = 2.5, 8.5 Hz, 2H, Ar-CH), 7.69 $(\mathrm{dd}, J=2.6,7.8 \mathrm{~Hz}, 2 \mathrm{H}, \mathrm{Ar}-\mathrm{CH}), 7.51$ (d, $J=7.8 \mathrm{~Hz}, 1 \mathrm{H}, \mathrm{Ar}-\mathrm{CH}), 7.23(\mathrm{~d}, J=2.8 \mathrm{~Hz}, 1 \mathrm{H}, \mathrm{Ar}-\mathrm{CH})$. ${ }^{13} \mathrm{C}$ NMR (DMSO-d6) $\delta 150.6(\mathrm{C}), 144.3(\mathrm{C}), 142.1(\mathrm{CH}), 139.6(\mathrm{C}), 136.0(\mathrm{C}), 130.3(\mathrm{CH}), 128.8$ $(\mathrm{CH}), 128.7(\mathrm{C}), 128.2(\mathrm{C}), 127.0(\mathrm{C}), 126.2(\mathrm{C}), 123.0(\mathrm{CH}), 121.9(\mathrm{CH}), 113.7(\mathrm{CH}), 112.9(\mathrm{C})$. HRMS: Calcd $[\mathrm{M}+\mathrm{H}]^{+}$for $\mathrm{C}_{16} \mathrm{H}_{10} \mathrm{~N}_{3} \mathrm{Cl}$ : 280.0643. Found: 280.0651 .

3-(Quinoxalin-2-yl)-1H-indole-5-carbonitrile (4i). This compound was obtained as pale yellow solid. Mp 331-335 ${ }^{\circ} \mathrm{C}$. IR (KBr, cm $\left.{ }^{-1}\right): 3439(\mathrm{NH}), 2220(\mathrm{CN}) .{ }^{1} \mathrm{H}$ NMR (DMSO-d6) $\delta 12.23$ (brs, 1H, NH), 9.20 (s, 1H, Ar-CH), 9.02 (s, 1H, Ar-CH), 7.94 (d, J = 7.8 Hz, 1H, Ar-CH), 7.65-7.57 (m, $3 \mathrm{H}, \mathrm{Ar}-\mathrm{CH}), 7.41$ (d, $J=7.8 \mathrm{~Hz}, 1 \mathrm{H}, \mathrm{Ar}-\mathrm{CH}), 7.31-7.29(\mathrm{~m}, 2 \mathrm{H}, \mathrm{Ar}-\mathrm{CH}) .{ }^{13} \mathrm{C}$ NMR (DMSO- $\left.d_{6}\right) \delta$ 154.7 (C), 151.9 (C), $138.6(\mathrm{C}), 135.4(\mathrm{CH}), 132.7$ (C), 130.8 (C), $129.2(\mathrm{CH}), 128.5(\mathrm{CH}), 126.0$ $(\mathrm{CH}), 123.9(\mathrm{CH}), 121.21(\mathrm{C}), 115.3(\mathrm{CH}), 114.0(\mathrm{CH}), 112.19(\mathrm{C}), 103.5(\mathrm{C})$. HRMS: Calcd $[\mathrm{M}+\mathrm{H}]^{+}$for $\mathrm{C}_{17} \mathrm{H}_{10} \mathrm{~N}_{4}: 271.0985$. Found: 271.0986 .

3-(6,7-Dichloroquinoxalin-2-yl)-1H-indole-5-carbonitrile (4j). This compound was obtained as bottle green powder like solid. $\mathrm{Mp}>300{ }^{\circ} \mathrm{C}$. IR $\left(\mathrm{KBr}, \mathrm{cm}^{-1}\right): 3303(\mathrm{NH}), 2228(\mathrm{CN}) .{ }^{1} \mathrm{H}$ NMR $\left(\mathrm{DMSO}-d_{6}\right) \delta 12.19$ (brs, 1H, NH), 9.10 (s, 1H, Ar-CH), 8.98 (s, 1H, Ar-CH), 8.26 (s, 1H, Ar-CH), 7.60-7.51 (m, 3H, Ar-CH), 7.33 (s, 1H, Ar-CH). ${ }^{13} \mathrm{C}$ NMR (DMSO-d6) $\delta 154.3(\mathrm{CH}), 152.7(\mathrm{C})$, $138.6(\mathrm{C}), 136.1(\mathrm{C}), 132.4(\mathrm{C}), 130.6(\mathrm{CH}), 129.3(\mathrm{C}), 128.5(\mathrm{CH}), 126.1(\mathrm{CH}), 125.4(\mathrm{CH}), 120.9$ $(\mathrm{CH}), 116.3(\mathrm{CN}), 113.8(\mathrm{CH}), 111.9(\mathrm{C}), 103.8(\mathrm{C})$. HRMS: Calcd $[\mathrm{M}+\mathrm{H}]^{+}$for $\mathrm{C}_{17} \mathrm{H}_{8} \mathrm{~N}_{4} \mathrm{Cl}_{2}$ : 339.0206. Found: 339.0208.

2-(5-Methoxy-2-methyl-1H-indol-3-yl)quinoxaline (4k). This compound was obtained as yellow powder. Mp 195-197 ${ }^{\circ} \mathrm{C}$. IR (KBr, cm $\left.{ }^{-1}\right): 3438(\mathrm{NH}) .{ }^{1} \mathrm{H}$ NMR (DMSO-d $) \delta 11.54$ (brs, 1H, NH), 9.21 (s, 1H, Ar-CH), 8.02-7.99 (m, 2H, Ar-CH), 7.80-7.77 (m, 2H, Ar-CH), 7.69 (dd, J = 3.1, 7.8 $\mathrm{Hz}, 1 \mathrm{H}, \mathrm{Ar}-\mathrm{CH}), 7.27$ (d, $J=7.8 \mathrm{~Hz}, 1 \mathrm{H}, \mathrm{Ar}-\mathrm{CH}), 6.78$ (dd, $J=3.1 \mathrm{~Hz}, 7.8 \mathrm{~Hz}, 1 \mathrm{H}, \mathrm{Ar}-\mathrm{CH}), 3.79$ $\left(\mathrm{s}, 3 \mathrm{H}, \mathrm{OCH}_{3}\right), 2.75\left(\mathrm{~s}, 3 \mathrm{H}, \mathrm{CH}_{3}\right) .{ }^{13} \mathrm{C} \mathrm{NMR}$ (DMSO-d $) \delta 155.0(\mathrm{C}), 151.9(\mathrm{C}), 145.5(\mathrm{CH}), 142.6$ (C), $139.6(\mathrm{C}), 139.5(\mathrm{C}), 130.6(\mathrm{CH}), 129.2(\mathrm{CH}), 128.9(\mathrm{CH}), 128.5(\mathrm{CH}), 112.2(\mathrm{CH}), 111.5(\mathrm{C})$, $109.7(\mathrm{C}), 102.8(\mathrm{CH}), 55.7\left(\mathrm{OCH}_{3}\right), 14.8\left(\mathrm{CH}_{3}\right)$. HRMS: Calcd $[\mathrm{M}+\mathrm{H}]^{+}$for $\mathrm{C}_{18} \mathrm{H}_{15} \mathrm{~N}_{3} \mathrm{O}: 290.1288$. Found: 290.1301.

2-(5-Methoxy-2-methyl-1H-indol-3-yl)-6,7-dimethylquinoxaline (4I). This compound was obtained as bright yellow powder. Mp 233-235 ${ }^{\circ} \mathrm{C}$. IR $\left(\mathrm{KBr}, \mathrm{cm}^{-1}\right): 3438(\mathrm{NH}) .{ }^{1} \mathrm{H}$ NMR (DMSO$\left.d_{6}\right) \delta 11.46$ (brs, 1H, NH), 9.08 (s, 1H, Ar-CH), 7.79 (s, 1H, Ar-CH), 7.75-7.65 (m, 2H, Ar-CH), 7.26 (d, $J=7.8 \mathrm{~Hz}, 1 \mathrm{H}, \mathrm{Ar}-\mathrm{CH}), 6.77$ (dd, $J=2.5 \mathrm{~Hz}, 7.8 \mathrm{~Hz}, 1 \mathrm{H}, \mathrm{Ar}-\mathrm{CH}), 3.78$ (s, 3H, OCH $)_{3}$, $2.72\left(\mathrm{~s}, 3 \mathrm{H}, \mathrm{CH}_{3}\right), 2.41\left(\mathrm{~s}, 6 \mathrm{H}, \mathrm{CH}_{3}\right) .{ }^{13} \mathrm{C} \mathrm{NMR}$ (DMSO-d6): $\delta 154.9(\mathrm{C}), 151.0(\mathrm{C}), 144.5(\mathrm{CH})$, $141.4(\mathrm{C}), 140.6(\mathrm{C}), 138.8(\mathrm{C}), 138.5(\mathrm{C}), 130.9$ (C), $128.2(\mathrm{CH}), 128.0(\mathrm{CH}), 127.9(\mathrm{C}), 112.1$ $(\mathrm{CH}), 111.3(\mathrm{CH}), 109.9(\mathrm{C}), 102.8(\mathrm{CH}), 55.8\left(\mathrm{OCH}_{3}\right), 20.3\left(\mathrm{CH}_{3}\right), 20.2\left(\mathrm{CH}_{3}\right), 14.7\left(\mathrm{CH}_{3}\right)$. HRMS: Calcd $[\mathrm{M}+\mathrm{H}]^{+}$for $\mathrm{C}_{20} \mathrm{H}_{19} \mathrm{~N}_{3} \mathrm{O}: 318.1601$. Found: 318.1613.

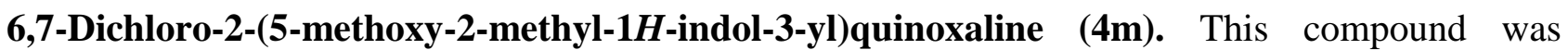
obtained as greenish crystalline solid. Mp 219-221 ${ }^{\circ} \mathrm{C}$. IR $\left(\mathrm{KBr}, \mathrm{cm}^{-1}\right): 3438(\mathrm{NH}) .{ }^{1} \mathrm{H}$ NMR (DMSO-d $)_{6} \delta 11.61$ (brs, 1H, NH), 9.15 (s, 1H, Ar-CH), 8.17 (s, 1H, Ar-CH), 8.14 (s, 1H, Ar-CH), $7.8(\mathrm{~s}, 1 \mathrm{H}, \mathrm{Ar}-\mathrm{CH}), 7.25$ (s, 1H, Ar-CH), 6.77 (s, 1H, Ar-CH), 3.79 (s, 3H, OCH $), 2.70(\mathrm{~s}, 3 \mathrm{H}$, 
$\mathrm{CH}_{3}$ ). ${ }^{13} \mathrm{C}$ NMR (DMSO- $\left.d_{6}\right) \delta 155.2(\mathrm{C}), 152.9(\mathrm{C}), 146.5(\mathrm{CH}), 141.6(\mathrm{C}), 140.6(\mathrm{C}), 138.2(\mathrm{C})$, $133.0(\mathrm{C}), 130.8(\mathrm{C}), 130.4(\mathrm{C}), 129.9(\mathrm{CH}), 129.5(\mathrm{CH}), 127.9(\mathrm{C}), 112.2(\mathrm{CH}), 111.7(\mathrm{CH}), 109.3$ (C), $103.3(\mathrm{CH}), 55.8\left(\mathrm{OCH}_{3}\right), 15.2\left(\mathrm{CH}_{3}\right)$. HRMS: Calcd $[\mathrm{M}+\mathrm{H}]^{+}$for $\mathrm{C}_{18} \mathrm{H}_{13} \mathrm{~N}_{3} \mathrm{Cl}_{2} \mathrm{O}: 358.0508$. Found: 358.0525 .

Methyl-2-(5-methoxy-2-methyl-1H-indol-3-yl)quinoxaline-6-carboxylate (4n). This compound was obtained as bright yellow solid. Mp 224-226 ${ }^{\circ} \mathrm{C}$. IR $\left(\mathrm{KBr}, \mathrm{cm}^{-1}\right): 3337(\mathrm{NH}), 1728(\mathrm{CO}) .{ }^{1} \mathrm{H}$ NMR (DMSO- $\left.d_{6}\right) \delta 11.68$ (brs, 1H, NH), 9.29 (s, 1H, Ar-CH), 8.49 (s, 1H, Ar-CH), 8.18 (d, J=8.0 $\mathrm{Hz}, 1 \mathrm{H}, \mathrm{Ar}-\mathrm{CH}$ ), 8.07 (d, $J=8.0 \mathrm{~Hz}, 1 \mathrm{H}, \mathrm{Ar}-\mathrm{CH}), 7.89$ (s, 1H, Ar-CH), 7.28 (d, $J=8.0 \mathrm{~Hz} .1 \mathrm{H}, \mathrm{Ar}-$ $\mathrm{CH}), 6.78(\mathrm{~d}, J=8.0 \mathrm{~Hz}, 1 \mathrm{H}, \mathrm{Ar}-\mathrm{CH}), 3.91\left(\mathrm{~s}, 3 \mathrm{H}, \mathrm{OCH}_{3}\right), 3.80\left(\mathrm{~s}, 3 \mathrm{H}, \mathrm{OCH}_{3}\right), 2.77\left(\mathrm{~s}, 3 \mathrm{H}, \mathrm{CH}_{3}\right)$. ${ }^{13} \mathrm{C}$ NMR (DMSO- $\left.d_{6}\right): \delta 166.2(\mathrm{C}=\mathrm{O}), 155.2(\mathrm{C}), 153.6(\mathrm{C}), 146.5$ (C), $145.1(\mathrm{C}), 141.0(\mathrm{C}), 138.4$ $(\mathrm{C}), 131.1(\mathrm{C}), 130.9(\mathrm{CH}), 129.6(\mathrm{C}), 128.0(\mathrm{CH}), 112.3(\mathrm{CH}), 111.9(\mathrm{CH}), 109.6(\mathrm{CH}), 103.3(\mathrm{C})$, $55.8\left(\mathrm{OCH}_{3}\right), 53.0\left(\mathrm{OCH}_{3}\right), 15.3\left(\mathrm{CH}_{3}\right)$. HRMS: Calcd $[\mathrm{M}+\mathrm{H}]^{+}$for $\mathrm{C}_{20} \mathrm{H}_{17} \mathrm{~N}_{3} \mathrm{O}_{3}: 348.1350$. Found: 348.1351 .

2-(5-Methoxy-2-methyl-1H-indol-3-yl)-6-nitroquinoxaline (4o). This compound was obtained as orange red solid. Mp 229-131 ${ }^{\circ} \mathrm{C}$. IR $\left(\mathrm{KBr}, \mathrm{cm}^{-1}\right): 3373(\mathrm{NH}) .{ }^{1} \mathrm{H}$ NMR (DMSO- $\left.d_{6}\right) \delta 11.76$ (brs, 1H, NH), 9.30 (s, $1 \mathrm{H}, \mathrm{Ar}-\mathrm{CH}), 8.66$ (s, $1 \mathrm{H}, \mathrm{Ar}-\mathrm{CH}), 8.37$ (d, $J=9.0 \mathrm{~Hz}, 1 \mathrm{H}, \mathrm{Ar}-\mathrm{CH}), 8.07$ (d, $J=$ $9.0 \mathrm{~Hz}, 1 \mathrm{H}, \mathrm{Ar}-\mathrm{CH}), 7.88(\mathrm{~s}, 1 \mathrm{H}, \mathrm{Ar}-\mathrm{CH}), 7.25(\mathrm{~d}, J=8.6 \mathrm{~Hz}, 1 \mathrm{H}, \mathrm{Ar}-\mathrm{CH}), 6.77$ (d, $J=8.6 \mathrm{~Hz}, 1 \mathrm{H}$, Ar-CH), 3.80 (s, 3H, $\mathrm{OCH}_{3}$ ), 2.76 (s, 3H, $\mathrm{CH}_{3}$ ); ${ }^{13} \mathrm{C}$ NMR (DMSO-d $) \delta 155.4(\mathrm{C}), 154.2(\mathrm{C}), 147.3$ $(\mathrm{CH}), 145.7(\mathrm{C}), 142.2(\mathrm{C}), 137.5(\mathrm{C}), 130.9(\mathrm{C}), 130.2(\mathrm{CH}), 128.1(\mathrm{C}), 125.1(\mathrm{CH}), 123.9(\mathrm{CH})$, 112.2 $(\mathrm{CH}), 111.9(\mathrm{CH}), 109.5(\mathrm{C}), 103.8(\mathrm{CH}), 55.8\left(\mathrm{OCH}_{3}\right), 15.4\left(\mathrm{CH}_{3}\right)$. HRMS: Calcd $[\mathrm{M}+\mathrm{H}]^{+}$ for $\mathrm{C}_{18} \mathrm{H}_{14} \mathrm{~N}_{4} \mathrm{O}_{3}: 335.1146$. Found: 335.1149 .

2-(1H-Indol-3-yl)quinoxaline-6-carbonitrile (4p). This compound was obtained as light yellow solid. Mp 297-299 ${ }^{\circ} \mathrm{C}$. IR $\left(\mathrm{KBr}, \mathrm{cm}^{-1}\right): 3438(\mathrm{NH}), 2221(\mathrm{CN}) .{ }^{1} \mathrm{H}$ NMR (DMSO- $\left.d_{6}\right) \delta 11.98$ (brs, $1 \mathrm{H}, \mathrm{NH}), 9.53(\mathrm{~s}, 1 \mathrm{H}, \mathrm{Ar}-\mathrm{CH}), 8.78-8.52$ (m, 2H, Ar-CH), 8.26-8.08 (m, 2H, Ar-CH), 7.56-7.50 (m, $2 \mathrm{H}, \mathrm{Ar}-\mathrm{CH}$ ), 7.42-7.23 (m, 2H, Ar-CH). ${ }^{13} \mathrm{C}$ NMR (DMSO- $\left.d_{6}\right) \delta 152.2(\mathrm{CH}), 145.8(\mathrm{CH}), 143.8$ (C), $139.1(\mathrm{C}), 137.7(\mathrm{C}), 133.4(\mathrm{C}), 130.3(\mathrm{CH}), 129.2(\mathrm{CH}), 128.7(\mathrm{CH}), 126.6(\mathrm{C}), 123.5(\mathrm{CH})$, 123.0 (CH), 121.6 (CH), 113.3 (CN), 112.7 (CH). HRMS: Calcd $[\mathrm{M}+\mathrm{H}]^{+}$for $\mathrm{C}_{17} \mathrm{H}_{10} \mathrm{~N}_{4}$ : 271.0985 . Found: 271.0986.

\section{Acknowledgements}

The authors are grateful to NIH (1RC2NS064950) for generous financial support.

\section{References}

1. Kaneko, C.; Katagiri, S. Asahi Glass Co. Ltd., Japan Kokai Tokkyo Koho Jp. 1998, 62, 207 (Chem.Abstr. 1998, 109, 231061)

2. Sarges, R.; Howard, H. R.; Browne, R. C.; Label, L. A.; Seymour, P. A. J. Med. Chem. 1990, $33,2240$. 
3. Kinashi, H.; Otten, S. L.; Dunkan, J. S.; Hutchinson, C. R. J. Antibiot. 1998, 41, 642.

4. Bryckaert, M. C.; Eldor, A.; Gazit, A.; Osherov, N.; Gilon, C.; Fontenay, M.; Levitzki, A.; Tobelem, G. Exp. Cell. Res. 1992, 199, 255.

5. Kovalenko, M.; Gazit, A.; Bohmer, C. R.; Rosman, C.; Ronnstrand, L.; Heldin, C. H.; Waltenberger, J.; Bohmer, F. D. Cancer Res. 1994, 54, 6106.

6. Spada, A. P.; Maguire, M. P.; Persons, P. E.; Myers, M. R. International Patent Application WO 92/20642, Nov 26, 1992. Ann drug report 1993, 588.

7. (a) Monge, A.; Palop, J. A.; Del Castillo, J. C.; Caldero, J. M.; Roca, J.; Romero, G.; Del Rio, J.; Lasheras, B. J. Med. Chem. 1993, 36, 2745. (b) Waring, M. J.; Makoff, A. Mol. Pharmacol. 1974, 10, 214. (c) For patent literature, see: Gunasekera, S. P.; Cross, S. S.; Kashman, Y.; Lui, M. S. Eur. Patent 272 810, 1998; Chem. Abstr. 1988, 109, 129417q.

8. (a) Garag, N. K.; Sarpong, R.; Stoltz, B. M. J. Am. Chem. Soc. 2002, 124, 13179. (b) Shaw, K. N. F.; cMillan, A.; Gudmundson, A. G.; Armstrong, M. D. J. Org. Chem. 1958, 23, 1171. (c) Hashem, M. A.; Sultan, I.; Hai, M. A. Indian J. Chem. Sec. B. 1998, $38,789$.

9. (a) Speeter, N. E.; Anthony, W. C. J. Am. Chem. Soc. 1954, 76, 6209. (b) Kharasch, M. S.; Kane, S. S.; Brown, H. C. J. Am. Chem. Soc. 1940, 62, 2242. (c) Brutcher, F. V.; Vanderwerff, W. D. J. Org.Chem. 1958, 23, 146. (d) Millich, F.; Becker, E. J. Org.Chem. 1958, 23, 1096. (e) Aubry, C.; Wilson, A. J.; Emmerson, D.; Murphy, E.; Chan, Y. Y.; Dickens, M. P.; Garcia, M. D.; Jenkins, P. R.; Mahale, S.; Chaudhuri, B. Bioorg. Med. Chem. 2009, 17, 6073.

10. Vermeulen, E. S.; van Smeden, M.; Schmidt, A. W.; Sprouse, J. S.; Wikstrom, H. V.; Grol, C. J. J. Med. Chem. 2004, 47, 5451.

11. Kuivila, H. G. J. Org. Chem. 1960, 25, 284.

12. (a) Kishi, Y.; Goto, T.; Inoue, S.; Siguira, S.; Kishimoto, H. Tetrahedron Lett. 1966, 7, 3445.

(b) Sarkis, G. Y.; Al-Azawe, S. J. Chem. Eng. 1973, 18, 102. (c) Gazit, A.; App, H.; McMahon, G.; Chen, J.; Levitzki, A.; Bohmer, F. D. J. Med. Chem. 1996, 39, 2170. 